\title{
\& Research Square

\section{Clinical Benefits of Early Concurrent use of Cryoprecipitate and Plasma Compared with Plasma only in Bleeding Trauma Patients: Analysis of the Trauma Quality Improvement Program Database}

\author{
Akira Endo \\ Tokyo Medical and Dental University: Tokyo Ika Shika Daigaku \\ Atsushi Senda \\ Tokyo Medical and Dental University: Tokyo Ika Shika Daigaku \\ Yasuhiro Otomo \\ Tokyo Medical and Dental University: Tokyo Ika Shika Daigaku \\ Raul Coimbra ( $\sim$ raulcoimbra62@yahoo.com ) \\ Riverside University Health System Medical Center
}

Research

Keywords: Polytrauma, resuscitation, hemorrhage shock, registry, transfusion, fibrinogen

Posted Date: August 23rd, 2021

DOI: https://doi.org/10.21203/rs.3.rs-815321/v1

License: (c) (i) This work is licensed under a Creative Commons Attribution 4.0 International License.

Read Full License 


\section{Abstract}

\section{Background:}

The effectiveness of cryoprecipitate in trauma has not been well-established. In addition, the benefits of cryoprecipitate might have been overestimated in previous studies since the difference in the total amount of administered clotting factors was not considered. This study aimed to evaluate the benefits of the concurrent use of cryoprecipitate in combination with fresh frozen plasma (FFP) during the resuscitation of bleeding trauma patients.

Methods: A retrospective cohort study using the American College of Surgeons Trauma Quality Improvement Program database was conducted. Patients who received $\geq 4$ units of packed red blood cells within the first 4 hours after arrival to the emergency department (ED) were dichotomized according to whether cryoprecipitate was used. The outcomes (primary: in-hospital mortality; secondary: 24-h mortality and incidence of complications) were compared using propensity score-matching analysis, in which the amount of total administered clotting factors was balanced.

Results: A total of 19,008 patients (Cryo+FFP group: 3825; FFP only group: 15,183) were eligible for analysis, of whom 3103 propensity score-matched pairs were generated. Significantly lower in-hospital mortality (1217 [39.2\%] patients in the Cryo+FFP group vs. 1295 [41.7\%] in the FFP only group; odds ratio [OR], 0.90; 95\% Cl, 0.81-1.00) and 24-h mortality (735 [23.7\%] vs. 886 [28.6\%]; OR, 0.78; 95\% Cl, 0.69-0.87) were observed in the Cryo+FFP group than in the FFP only group; no significant difference was observed in the incidence of complications.

Conclusions: Cryoprecipitate use combined with FFP was significantly associated with reduced mortality in bleeding trauma patients after adjustments were made for the dose of administered clotting factors. Future randomized controlled trials are warranted to confirm our results.

\section{Introduction}

Hemorrhage is the leading cause of early death in severe trauma [1, 2]. In addition to hemostatic procedures, an appropriate transfusion strategy is essential in the resuscitation of bleeding trauma patients [3, 4]. However, there is still debate about what constitutes an optimal transfusion strategy [5]. Although the importance of clotting factors supplementation, including fibrinogen, in hemostatic resuscitation has been well-recognized [3-6], clinical evidence supporting the effectiveness of the use of clotting factor preparation has been scarce $[7,8]$. Hence, compared to the optimal blood products ratio, the use of coagulation factor concentrates has not been standardized [9].

Cryoprecipitate is prepared by concentrating fresh frozen plasma (FFP), in which high concentrations of clotting factors, including fibrinogen and coagulation factors VIII, XIII, and von Willebrand factor (VWF) are present [10]. Although cryoprecipitate was originally developed to treat patients with congenital coagulation protein deficiencies [11], due to the development of virus-inactivated coagulation factor 
concentrates and recombinant factor preparations, its use for these patients is currently not very common. Recently, cryoprecipitate has been mainly used for patients with acquired coagulopathy, which is observed in trauma patients [12].

However, clinical evidence supporting the use of cryoprecipitate in trauma is scarce. A previous small randomized controlled trial [13] demonstrated the feasibility of cryoprecipitate use in bleeding trauma patients. Further, a previous retrospective cohort study analyzed a large-scale trauma registry and showed a significant reduction in mortality in patients who received cryoprecipitate [14]. However, because the total administered amount of clotting factors was not balanced between the two groups in the latter large-scale retrospective cohort study, the benefits of cryoprecipitate might have been overestimated by the total amount of administered clotting factors.

Since trauma patients requiring hemostatic resuscitation usually experience a massive loss of intravascular volume, the administration of FFP in sufficient doses without cryoprecipitate might be enough to replace the loss of clotting factors [15]. In the present study, we aimed to evaluate whether early use of cryoprecipitate in addition to FFP during the resuscitation of bleeding trauma patients has a beneficial effect on outcomes compared to FFP only, taking into account the total amount of clotting factors given by FFP and cryoprecipitate.

\section{Methods}

\section{Design and Setting}

This retrospective cohort study analyzed data abstracted from the Trauma Quality Improvement Program (TQIP) database between 2014 and 2017. The TQIP database is a subset of the National Trauma Databank of the American College of Surgeons Committee on Trauma, which stores data of patients who experienced severe injury, defined by an abbreviated injury scale (AIS) $\geq 3$ and aged 16 years or older. Trained registrars collected patient information on over 100 variables, as well as information on the treating hospital. Details in the TQIP database are available at https://www.facs.org/qualityprograms/trauma/tqp/center-programs/tqip.

The present study complied with the principles of the 1964 Declaration of Helsinki and its later amendments. The study and its protocols complied with the institutional review board of the Riverside University Health System-Comparative Effectiveness and Clinical Outcomes Research Center (Approval number: 1636962). The requirement for informed consent for each patient was waived based on the use of anonymized patient data.

\section{Study population}

Patients in the TQIP database who met the following criteria were included in the study: (1) patients directly transported from the scene of injury, (2) patients who received 4 units or more of packed red blood cells ( $\mathrm{pRBCs}$ ) within 4 hours of admission, and (3) patients who received 1 unit or greater of FFP 
within 4 hours of admission. In addition, patients aged 90 years or older, those with unsalvageable injury defined by AIS = 6 in any body region, patients without signs of life (SOL) upon arrival at the emergency department (ED), or those with missing values for the variables listed in the aforementioned exclusion criteria or outcomes were also excluded after multiple imputations. In addition, we excluded patients who received more than 100 units of pRBCs, FFP, platelets, or cryoprecipitate in the first 4 hours of ED arrival, considering the potential inaccuracy of recorded data that could lead to skewed causal inference.

Patients eligible for analysis were divided into two groups: the Cryo + FFP group and the FFP only group according to whether cryoprecipitate was administered within the first 4 hours of ED arrival.

\section{Definition and Outcomes}

The concentration ratios of clotting proteins in the cryoprecipitate compared to FFP, to some extent, varies according to the type of clotting factor, the blood donors, and the manufacturing process [16]. Thus, determining a uniform conversion ratio for the total clotting factors amount between cryoprecipitate and FFP is challenging. In the present study, because cryoprecipitate generally has a 3-fold higher fibrinogen concentration than FFP [16], and since the cryoprecipitate is mainly used for fibrinogen supplementation [17], the amount of clotting factors available in the cryoprecipitate was estimated based on the assumption that the concentration of clotting factors is three-fold higher in cryoprecipitate than in FFP.

The primary outcome was in-hospital mortality. Secondary outcomes were defined as in-hospital death within $24 \mathrm{~h}$ after ED arrival and the incidence of complications during hospital stay. Complications were defined as a composite of cardiac arrest, unplanned intubation, deep vein thrombosis, pulmonary embolism, myocardial infarction, stroke, acute kidney injury, sepsis, central line-associated bloodstream infection, ventilator-associated pneumonia, catheter-associated urinary tract infection, and organ space surgical site infection.

\section{Variables}

The following variables were extracted from the TQIP database: injury mechanism (blunt, penetrating, or unspecified/others); age; sex; bleeding disorders including anticoagulants use; vital signs (systolic blood pressure and respiratory rate) upon ED arrival; the Glasgow Coma Scale (GCS) upon ED arrival; presence or absence of SOL upon ED arrival; AIS in each body region (head, face, neck, thorax, abdomen, spine, upper extremity, pelvis and lower extremity, and surface); Injury Severity Score (ISS); units and volume of pRBC, FFP, platelets, and cryoprecipitate administered within the first 4 hours after ED arrival; conversion ratio between transfusion unit and volume $(\mathrm{mL})$ in each facility; length of hospital stay (days and minutes); incidence of complications during hospital stay; and survival status at hospital discharge.

\section{Statistical analysis}

The multiple imputation method, using chained equations with 10 iterations and the creation of 15 datasets, was used to treat missing values based on the assumption of missing at random in the missing mechanism, as done in previous studies using the TQIP database $[18,19]$. 
We used the propensity score-matching method as the primary analysis [20], considering the nature of non-randomized data where patient characteristics were not equally distributed. In this analysis, a logistic regression model was applied to estimate the propensity score for predicting cryoprecipitate use within the first 4 hours of ED arrival for each patient. The following variables were selected a priori, considering the clinical importance and subject matter knowledge [21], and incorporated into the model: age; sex; presence or absence of bleeding disorders; mechanism of injury (blunt, penetrating, or others); systolic blood pressure, respiratory rate, and GCS upon ED arrival; the highest AIS score in each body region; ISS; and the volume of pRBCs, platelets, and total clotting factors administered within the first 4 hours of ED arrival. The total amount of clotting factors administered within the first $4 \mathrm{~h}$ of ED arrival was calculated using the FFP and cryoprecipitate volume and presented as FFP standard. The predictive accuracy of the logistic regression model, using the aforementioned variables for in-hospital mortality was assessed using the $C$ statistic. Through the 1:1 matching process, pairs from the Cryo + FFP and FFP only groups were generated. Match balance between the two groups was assessed using the absolute standardized mean difference (ASMD), where values of less than 0.1 were considered acceptable [22]. The caliper width was set as the standard deviation of the logit-transformed propensity score multiplied by 0.01 to achieve a good balance between the two groups [23]. The chi-square test was used for intergroup comparison in the propensity score-matched cohort.

In addition, as an exploratory analysis, cumulative incidence curves for in-hospital mortality in the propensity score-matched subjects were constructed. Finally, the Fine and Gray test [24] was used to estimate the subdistribution hazard ratio for in-hospital mortality, considering the competing risk between patient mortality and survival discharge as TQIP database is an inpatient database.

Concerning the sensitivity analysis of the conversion ratio between cryoprecipitate and FFP, we performed propensity score matching analysis. The amounts of clotting factors in the cryoprecipitate were estimated based on the assumption that clotting proteins are concentrated 5 -fold and 10 -fold, respectively, in the cryoprecipitate compared to FFP. In addition, we performed a sensitivity analysis only for complete cases, using the same method as the primary analysis, as validation of the multiple imputation method.

Descriptive statistics were reported as counts (percentages) for categorical variables and medians (25th -75th percentiles) for numeric or ordered variables after pooling all the imputed datasets into one dataset. Predictive statistics were reported as odds ratios (ORs) and $95 \%$ confidence intervals (Cls) by integrating across the imputed datasets, based on Rubin's rule [25]. The level of significance was defined as twosided $p<.05$ for all statistical analyses. All analyses were performed using R 4.0.4 (R Foundation for Statistical Computing, Vienna, Austria) with add-on packages of "mice [26]" for multiple imputations, "Matching [27]" for propensity score matching, and "cmprsk [28]" for the Fine and Gray test.

\section{Results}


The patient selection flow diagram is shown in Fig. 1. A total of 19,008 patients (Cryo + FFP group: 3825; FFP only group: 15,183 ) were eligible for analysis; median age (25th - 75th percentiles) was 37 (25-54), and 14,696 patients $(77.3 \%)$ were male. The characteristics of the raw data, including the proportion of missing values, are shown in eTable 1 in the Supplement. The baseline characteristics of the overall and propensity score-matched cohorts after multiple imputations are presented in Table 1. In the overall cohort, in-hospital mortality in the Cryo + FFP and FFP only groups was 1620 (42.4\%) and 4410 (29.0\%), respectively. The 24-h mortality was observed in 1005 (26.3\%) and 2641 (17.4\%), and incidence of complications was observed in 1495 (39.1\%) and 4570 (30.1\%). The median length of hospital stay (25th - 75th percentiles) in the overall cohort was 12 days (2-27) in the Cryo + FFP group and 12 days (4-24) in the FFP only group. In the overall cohort, significantly higher in-hospital mortality, 24-h mortality, and incidence of complications were observed in the Cryo + FFP group compared to the FFP only group (OR, $1.79 ; 95 \% \mathrm{Cl}, 1.67-1.93, \mathrm{OR}, 1.69 ; 95 \% \mathrm{Cl}, 1.56-1.84$, and $\mathrm{OR}, 1.49 ; 95 \% \mathrm{Cl}, 1.38-1.60$ ). 
Table 1

Baseline characteristics of the multiply imputed dataset before and after propensity score matching

Unmatched cohort

Variables

Number of subjects, $\mathrm{n}$

Age (years)

Sex, Male, $\mathrm{n}(\%)$

Bleeding disorder, $\mathrm{n}(\%)$

Mechanism of injury, $\mathrm{n}(\%)$

Blunt

Penetrating

Other/unspecified

Systolic blood pressure upon ED

arrival, $\mathrm{mmHg}$

Respiratory rate upon ED arrival, breaths/min

Glasgow coma scale upon ED arrival

Highest AIS score per body region

Head

$0[0,3]$

$0[0,3]$

$0[0,1]$

$0[0,1]$

$0[0,0]$

$0[0,0]$

$3[0,4]$

$3[0,4]$

$3[0,4]$

$0[0,2]$

Spine

Abdomen

$2[0,4]$

$0[0,2]$

0.05
Matched cohort

ASMD

Cryo +
FFP
group

FFP only group

ASMD

$3103 \quad 3103$

0.15

$35[24$,

$34[24$

0.04

$50]$

$\begin{array}{llll}0.06 & 2450 & 2463 & 0.01 \\ & (79.0) & (79.4) & \end{array}$

$0.07 \quad 79(2.5) \quad 64(2.1) \quad 0.03$

0.14

0.04

$1854 \quad 1799$

(59.7) (58.0)

$1242 \quad 1293$

(40.0) (41.7)

$7(0.2) \quad 11(0.4)$

$104 \quad 102[80,0.03$

$[80,129]$

Abbreviations: SOL, signs of life; ED, emergency department; FFP, fresh frozen plasma; Cryo, cryoprecipitate.

Abbreviations: FFP, fresh frozen plasma; Cryo, cryoprecipitate. 


\begin{tabular}{|c|c|c|c|c|c|c|}
\hline \multirow[b]{2}{*}{ Upper extremities } & \multicolumn{2}{|c|}{ Unmatched cohort } & \multicolumn{4}{|c|}{ Matched cohort } \\
\hline & $0[0,2]$ & $0[0,2]$ & 0.04 & $0[0,2]$ & $0[0,2]$ & 0.01 \\
\hline Pelvis and lower extremities & $2[0,3]$ & $2[0,3]$ & 0.01 & $2[0,3]$ & $2[0,3]$ & 0.01 \\
\hline Skin/Superficial & $0[0,0]$ & $0[0,0]$ & 0.02 & $0[0,0]$ & $0[0,0]$ & 0.01 \\
\hline Injury severity score & $\begin{array}{l}29[22, \\
41]\end{array}$ & $\begin{array}{l}26[17, \\
36]\end{array}$ & 0.27 & $\begin{array}{l}29[21, \\
41]\end{array}$ & $\begin{array}{l}30[22, \\
41]\end{array}$ & 0.07 \\
\hline \multicolumn{7}{|l|}{$\begin{array}{l}\text { Transfusion volume within the } \\
\text { first } 4 \text { hours of ED arrival, } \mathrm{mL}\end{array}$} \\
\hline Red Blood Cells & $\begin{array}{l}4200 \\
{[2450} \\
7000]\end{array}$ & $\begin{array}{l}2100 \\
{[1400} \\
3150]\end{array}$ & 0.82 & $\begin{array}{l}3575 \\
{[2100} \\
5700]\end{array}$ & $\begin{array}{l}3600 \\
{[2100} \\
5700]\end{array}$ & 0.01 \\
\hline Plasma* & $\begin{array}{l}2400 \\
{[1250} \\
4000]\end{array}$ & $\begin{array}{l}1000 \\
{[600,} \\
1750]\end{array}$ & 0.52 & $\begin{array}{l}2000 \\
{[1200} \\
3214]\end{array}$ & $\begin{array}{l}2500 \\
{[1500} \\
3840]\end{array}$ & 0.29 \\
\hline Platelets & $\begin{array}{l}500 \\
{[250} \\
750]\end{array}$ & $\begin{array}{l}200[0, \\
300]\end{array}$ & 0.58 & $\begin{array}{l}400 \\
{[238,} \\
700]\end{array}$ & $\begin{array}{l}300 \\
{[200} \\
600]\end{array}$ & 0.02 \\
\hline $\begin{array}{l}\text { Cryoprecipitate volume within the } \\
\text { first } 4 \text { hours of ED arrival** } \mathrm{mL}\end{array}$ & $\begin{array}{l}170 \\
{[100,} \\
300]\end{array}$ & $0[0,0]$ & 1.67 & $\begin{array}{l}150 \\
{[100,} \\
250]\end{array}$ & $0[0,0]$ & 1.68 \\
\hline $\begin{array}{l}\text { Clotting factors volume within the } \\
\text { first } 4 \text { hours of ED arrival, } \mathrm{mL}\end{array}$ & $\begin{array}{l}3060 \\
{[1830} \\
4850]\end{array}$ & $\begin{array}{l}1040 \\
{[600,} \\
1785]\end{array}$ & 1.06 & $\begin{array}{l}2600 \\
{[1610} \\
3897]\end{array}$ & $\begin{array}{l}2500 \\
{[1500,} \\
3840]\end{array}$ & 0.02 \\
\hline \multicolumn{7}{|c|}{ Numeric variables are expressed as median [25th -75 th percentiles]. } \\
\hline \multicolumn{7}{|c|}{$\begin{array}{l}\text { Abbreviations: Cryo, cryoprecipitate; FFP, fresh frozen plasma; ASMD, Absolute Standardized mean } \\
\text { difference; ED, emergency department. }\end{array}$} \\
\hline \multicolumn{7}{|c|}{$\begin{array}{l}\text { Clotting factors volume was estimated based on the assumption that clotting factors are } \\
\text { concentrated by 3-fold in cryoprecipitate compared to FFP and showed as FFP standard. }\end{array}$} \\
\hline \multicolumn{7}{|c|}{ *These variables were not included in the model for propensity score estimation. } \\
\hline \multicolumn{7}{|c|}{$\begin{array}{l}\text { Abbreviations: SOL, signs of life; ED, emergency department; FFP, fresh frozen plasma; Cryo, } \\
\text { cryoprecipitate. }\end{array}$} \\
\hline
\end{tabular}

(Table 1 here)

The variables used for propensity score estimation had high accuracy in predicting in-hospital mortality, with a $C$-statistic of 0.84 . Through the matching process, 3103 propensity score-matched patient pairs were generated. All the ASMD values used for matching were lower than 0.1 , indicating a good balance in the propensity score-matched cohort. The results of the propensity score-matching analysis are 
summarized in Table 2. Among the propensity score-matched subjects, in-hospital mortality in the Cryo + FFP and FFP only groups was 1217 (39.2\%) patients and 1295 (41.7\%), respectively. The 24-h mortality was observed in $735(23.7 \%)$ and $886(28.6 \%)$, and complications were observed in $1143(36.8 \%)$ and 1191 (38.4\%), respectively. The median length of hospital stay (25th - 75th percentiles) was 13 days (227 ) in the Cryo + FFP group and 11 days (1-26) in the FFP only group, respectively. In the propensity score-matched cohort, significantly lower in-hospital mortality and 24-h mortality were observed in the Cryo + FFP group compared to the FFP only group (OR, 0.90; $95 \% \mathrm{Cl}, 0.81-1.00$ and $\mathrm{OR}, 0.78 ; 95 \% \mathrm{Cl}$, $0.69-0.87$ ); however, no significant differences were observed in the incidence of complications (OR, $0.94 ; 95 \% \mathrm{Cl}, 0.84-1.04)$.

Table 2

Effect of cryoprecipitate compared to FFP only on the outcomes (multiple imputation cohort)

\begin{tabular}{|lll|}
\hline \multirow{2}{*}{ Outcomes } & \multicolumn{2}{l|}{ Odds ratio (95\% confidence intervals) } \\
\cline { 2 - 3 } & Overall cohort & Propensity score-matched cohort \\
\hline In-hospital mortality, $\mathrm{n}(\%)$ & $1.79(1.67-1.93)$ & $0.90(0.81-1.00)$ \\
\hline 24-h mortality, $\mathrm{n}(\%)$ & $1.69(1.56-1.84)$ & $0.78(0.69-0.87)$ \\
\hline Complications, $\mathrm{n}(\%)$ & $1.49(1.38-1.60)$ & $0.94(0.84-1.04)$ \\
\hline Abbreviation: FFP, fresh frozen plasma. & \\
\hline
\end{tabular}

Figure 2 shows the cumulative incidence curve in the propensity score-matched subjects for in-hospital mortality until 30 days after admission. The Fine and Gray test revealed significantly lower in-hospital mortality in the Cryo + FFP group than in the FFP only group (subdistribution hazard ratio $0.90 ; 95 \% \mathrm{Cl}$, 0.84-0.97).

The sensitivity analyses using the conversion ratios of 5 -fold and 10 -fold, instead of the 3 -fold, in clotting factors between cryoprecipitate and FFP showed similar results as observed in the primary analyses (eTables 2 and 3 in the Supplement). In addition, the analysis including all complete cases achieved similar results (eTables 4 and 5 in the Supplement).

\section{Discussion}

In the present study, we evaluated the survival benefit of cryoprecipitate use in the resuscitation phase of trauma patients requiring massive transfusion, in which the amounts of administered clotting factors were considered. The results suggested that the concurrent use of cryoprecipitate and FFP is more beneficial than the administration of FFP alone when supplementing the same amounts of clotting factors. Although significantly higher mortality was observed in the Cryo + FFP group in the overall cohort (not the propensity score-matched cohort), cryoprecipitate is generally used for more severe patients requiring a larger amount of transfusion, as shown in Table 1. After the appropriate case-mix adjustment, 
the potential advantage of early administration of cryoprecipitate was observed. From the cumulative incidence curve, the difference in mortality was large in the first 1 to 2 days and then maintained, suggesting that the hemostatic effect mainly caused the observed benefit.

The concentration ratios of clotting proteins in cryoprecipitate, compared to FFP, varied according to the type of clotting factor. For example, the concentrations of fibrinogen, factor VIII, and VWF have been estimated to be approximately 3,8 , and 12 times that of FFP, respectively, when cryoprecipitate is processed through blood banks in the United States. However, the concentration of clotting factors varies depending, to some extent, on the blood donors [16]. In the present study, we assumed a uniform conversion ratio between cryoprecipitate and FFP; however, the non-uniformity of concentration ratios in each cryoprecipitate product and the effects of clotting factors other than fibrinogen should also be considered. Although we also explored differences in outcomes according to different conversion ratios, assumptions based on a conversion using a certain ratio would not be the optimal approach. However, since a large-scale study evaluating the effectiveness of cryoprecipitate considering the amount of administered clotting factors has not been reported, the present study results provide significant clinical evidence on this aspect before large-scale randomized controlled trials are conducted.

Although the granular mechanism of cryoprecipitate could not be discussed from this registry-based analysis, there were several possible reasons for the significantly lower mortality rate observed in patients who received cryoprecipitate. If clotting factors are administered only with the purpose of supplementation in patients sustaining hemorrhagic shock, transfusion of whole blood or blood components with a 1:1:1 ratio of pRBC:FFP:platelets would suffice. However, in cases of severe injury, the presence of injury-induced coagulopathy, in addition to hemorrhage and blood dilution due to fluid resuscitation, is also common $[29,30]$. The results of this study have highlighted the benefits of cryoprecipitate administered as a treatment against trauma-induced coagulopathy, which accompanies the dysregulated fibrinolytic system and a relative deficiency in clotting factors observed in these patients. Furthermore, significant fluid overload is inevitable when administering large amounts of clotting factors by infusion of FFP only, which has been reported to have a negative impact on trauma patient outcomes [31-33]. Although the information on the administered fluid volume was not available in the TQIP database, appropriate volume management might have been achieved by administering concentrated clotting factors using cryoprecipitate. The RETIC trial [34] showed that the need for rescue therapies significantly decreased in patients who received clotting factor concentrates, primarily fibrinogen concentrate, compared to patients who received FFP only. We also evaluated the incidence of complications in the present study; however, no significant differences were observed between groups. Although a survivor bias was unavoidable in assessing this outcome measure, it works in an unfavorable direction in patients who survive longer, suggesting that cryoprecipitate could improve patient survival without increasing complications. The immunomodulatory effects of plasma also might have affected patient outcomes. Plasma and cryoprecipitate made from plasma contain various important proteins such as endothelial glycocalyx, fibronectin, and platelet microparticles, in addition to clotting factors, which have important biologic functions and are said to regulate vascular permeability [35, 36]. Although the concentrations of these immunomodulatory proteins in cryoprecipitate have not been well-studied, 
potential differences in the amount of administered plasma proteins between cryoprecipitate and FFP could be a possible explanation for the observed differences in the outcomes.

Clinical evidence for the comparative effectiveness of cryoprecipitate and other clotting factor preparations has been limited in bleeding patients [37,38]. A theoretical advantage of cryoprecipitate use is that it contains a variety of important coagulation factors. It has been reported that the levels of VWF and factor XIII, which play critical roles in primary and secondary hemostasis, are significantly decreased in trauma patients who experience major bleeding [39]. Moreover, fibrinogen concentrate manufacturing costs are, on average, 3 to 4 times higher than that of cryoprecipitate preparation [40]. However, there have also been concerns raised relative to the risk of viral infection using cryoprecipitate preparations. As opposed to fibrinogen concentrate, which is virally inactivated, the safety of cryoprecipitate use is still of concern, although several efforts for pathogen reduction have been made [12]. Furthermore, cryoprecipitate requires a thawing time of approximately 17 to $20 \mathrm{~min}$, in contrast to fibrinogen concentrate which can be stored in the ED at room temperature, allowing prompt administration when needed. Further clinical evidence is required to establish a next-stage optimal management strategy against trauma-induced coagulopathy in patients who require massive transfusions.

\section{Strength and Limitations}

This study had several strengths. First, we analyzed the large-scale trauma registry collecting real-world data of North America in which many patients who received massive transfusions were included. The amount of administered transfusion components and cryoprecipitate within the first $4 \mathrm{~h}$ were recorded in the database, which enabled us, for the first time, to evaluate the comparative benefits of cryoprecipitate in addition to FFP in hemostatic resuscitation. However, several limitations must be acknowledged. The issue of residual confounding was inevitable due to the retrospective nature of the study. Information on the laboratory test results, details about the time-course in the ED, use of tranexamic acid, and detailed hemostatic procedures were not available in the TQIP database. Also, the information on the administered fluid volume was unavailable in the TQIP database, which prevented assessing the dilutional effect. The use of fibrinogen concentrate could not be identified because the procedure code was not defined in the International Classification of Diseases 10th Revision before 2016, although a small amount of clotting factors in blood products was accounted for by matching administered pRBCs and platelets volume. The guideline for massive transfusion by the American College of Surgeons suggested using cryoprecipitate according to viscoelastic coagulation measures [41]; however, information on the use of the test was also unavailable. Although many patients were evaluated, generalizability was limited because the TQIP is a trauma database in the USA, exckuding patients younger than 16 years and older than 90 years. The accuracy and missing data in the database could also be considered a limitation. As discussed above, making assumptions about the conversion ratio between cryoprecipitate and FFP at a certain level would not be an optimal approach. Finally, the hemostatic resuscitation strategy was not uniform across trauma centers; each center treated patients with different strategies without consistency. Hospitals with well-established transfusion protocols, including the use of cryoprecipitate, might have achieved better outcomes. Due to the lack of information 
on the unique identifier of a treating hospital, the model considering the hospital-level clustering could not be used in our analysis.

\section{Conclusions}

The administration of cryoprecipitate was significantly associated with reduced in-hospital mortality in trauma patients requiring massive transfusion after adjustment for the amount of administered clotting factors. Future randomized controlled trials are warranted to confirm our results.

\section{List Of Abbreviations}

AIS, abbreviated injury scale; ASMD, absolute standardized mean difference; $\mathrm{Cl}$, confidence interval; ED, emergency department; FFP, fresh frozen plasma; GCS, Glasgow coma scale; ISS, injury severity score; OR, odds ratio; pRBC, packed red blood cell; SOL, signs of life; TQIP, Trauma Quality Improvement Program; vWF, von Willebrand factor.

\section{Declarations}

Ethics approval and consent to participate

The study and its protocols were approved by the institutional review committee of Riverside University Health System - Comparative Effectiveness and Clinical Outcomes Research Center. The requirement for informed consent for each patient was waived based on the use of anonymized patient and hospital data.

Consent for publication

Not applicable

Availability of data and material

All data used in the present study are available from the American College of Surgeons Trauma Quality Improvement Program.

Competing interests

The authors declare that they have no competing interests.

\section{Funding}

The authors have no conflicts of interest and no source of funding to declare. 
A.E., A.S., R.C., and Y.O contributed to project development and study design.

M.K., A.S., and R.C. contributed to data collection and interpretation of data.

A.E. and A.S. performed data analysis.

A.E. and R.C. wrote the manuscript.

R.C., A.S., and Y.O. critically edited and revised the manuscript.

\section{References}

1. Koh EY, Oyeniyi BT, Fox EE, Scerbo M, Tomasek JS, Wade CE, et al. Trends in potentially preventable trauma deaths between 2005-2006 and 2012-2013. Am J Surg. 2019;218:501-6.

2. Eastridge BJ, Holcomb JB, Shackelford S. Outcomes of traumatic hemorrhagic shock and the epidemiology of preventable death from injury. Transfusion. 2019;59:1423-8.

3. Holcomb JB, Tilley BC, Baraniuk S, Fox EE, Wade CE, Podbielski JM, et al. Transfusion of plasma, platelets, and red blood cells in a 1:1:1 vs a 1:1:2 ratio and mortality in patients with severe trauma: the PROPPR randomized clinical trial. JAMA. 2015;313:471-82.

4. Endo A, Shiraishi A, Fushimi K, Murata K, Otomo Y. Outcomes of patients receiving a massive transfusion for major trauma. Br J Surg. 2018;105:1426-34.

5. McQuilten ZK, Crighton G, Brunskill S, Morison JK, Richter TH, Waters N, et al. Optimal dose, timing and ratio of blood products in massive transfusion: Results from a systematic Review. Transfus Med Rev. 2018;32:6-15.

6. Stinger HK, Spinella PC, Perkins JG, Grathwohl KW, Salinas J, Martini WZ, et al. The ratio of fibrinogen to red cells transfused affects survival in casualties receiving massive transfusions at an army combat support hospital. J Trauma. 2008;64:79-85.

7. Novak A, Stanworth SJ, Curry N. Do we still need cryoprecipitate? Cryoprecipitate and fibrinogen concentrate as treatments for major hemorrhage - How do they compare? Expert Rev Hematol. 2018;11:351-60.

8. Winearls J, Campbell D, Hurn C, et al. Fibrinogen in traumatic haemorrhage: A narrative review. Injury. 2017;48:230-42.

9. Winearls J, Campbell D, Hurn C, Furyk J, Ryan G, Trout M, et al. Damage control resuscitation in patients with severe traumatic hemorrhage: A practice management guideline from the Eastern Association for the Surgery of Trauma. J Trauma Acute Care Surg. 2017;82:605-17.

10. Callum JL, Karkouti K, Lin Y. Cryoprecipitate: the current state of knowledge. Transfus Med Rev. 2009;23:177-88.

11. Cohn EJ, Strong LE, Hughes WL, Mulford DJ, Ashworth JN, Melin M, et al. Preparation and properties of serum and plasma proteins; a system for the separation into fractions of the protein and lipoprotein components of biological tissues and fluids. J Am Chem Soc. 1946;68:459-75. 
12. Nascimento B, Goodnough LT, Levy JH. Cryoprecipitate therapy. Br J Anaesth. 2014;113:922-34.

13. Curry N, Rourke C, Davenport R, Beer S, Pankhurst L, Deary A, et al. Early cryoprecipitate for major haemorrhage in trauma: a randomised controlled feasibility trial. Br J Anaesth. 2015;115:76-83.

14. Ditillo M, Hanna K, Castanon L, Zeeshan M, Kulvatunyou N, Tang A, et al. The role of cryoprecipitate in massively transfused patients: Results from the Trauma Quality Improvement Program database may change your mind. J Trauma Acute Care Surg. 2020;89:336-43.

15. Weymouth W, Long B, Koyfman A, Winckler C. Whole blood in trauma: A review for emergency clinicians. J Emerg Med. 2019;56:491-8.

16. Caudill JS, Nichols WL, Plumhoff EA, Schulte SL, Winters JL, Gastineau DA, et al. Comparison of coagulation factor XIII content and concentration in cryoprecipitate and fresh-frozen plasma. Transfusion. 2009;49:765-70.

17. Sparrow RL, Simpson RJ, Greening DW. A protocol for the preparation of cryoprecipitate and cryodepleted plasma for proteomic Studies. Methods Mol Biol. 2017;1619:23-30.

18. Butler EK, Mills BM, Arbabi S, Bulger EM, Vavilala MS, Groner JI, et al. Association of blood component ratios with 24-hour mortality in injured children receiving massive transfusion. Crit Care Med. 2019;47:975-83.

19. Endo A, Kojima M, Hong ZJ, Otomo Y, Coimbra R. Open-chest versus closed-chest cardiopulmonary resuscitation in trauma patients with signs of life upon hospital arrival: a retrospective multicenter study. Crit Care. 2020;24:541.

20. D'Agostino RB Jr. Propensity score methods for bias reduction in the comparison of a treatment to a non-randomized control group. Stat Med. 1998;17:2265-81.

21. Boyd CR, Tolson MA, Copes WS. Evaluating trauma care: the TRISS method. Trauma Score and the Injury Severity Score. J Trauma. 1987;27:370-8.

22. Austin PC. Balance diagnostics for comparing the distribution of baseline covariates between treatment groups in propensity-score matched samples. Stat Med. 2009;28:3083-107.

23. Lunt M. Selecting an appropriate caliper can be essential for achieving good balance with propensity score matching. Am J Epidemiol. 2014;179:226-35.

24. Fine JP, Gray RJ. A proportional hazards model for the subdistribution of a competing risk. J Am Stat Assoc. 1999;94:496-509.

25. Rubin DB. Multiple imputation for nonresponse in surveys. New York: John Wiley; 1987.

26. Zhang Z. Multiple imputation with multivariate imputation by chained equation (MICE) package. Ann Transl Med. 2016;4:30.

27. Sekhon JS. Multivariate and propensity score matching software with automated balance optimization: the matching package for R. J Stat Softw. 2011;42:1-52.

28. Gray B. cmprsk. Subdistribution analysis of competing risks. https://cran.rproject.org/web/packages/cmprsk/index.html. Published 2020. Accessed March 6, 2021. 
29. Chang R, Cardenas JC, Wade CE, Holcomb JB. Advances in the understanding of trauma-induced coagulopathy. Blood. 2016;128:1043-9.

30. Gando S, Otomo Y. Local hemostasis, immunothrombosis, and systemic disseminated intravascular coagulation in trauma and traumatic shock. Crit Care. 2015;19:72.

31. Bosboom JJ, Klanderman RB, Zijp M, Hollmann MW, Veelo DP, Binnekade JM, et al. Incidence, risk factors, and outcome of transfusion-associated circulatory overload in a mixed intensive care unit population: A nested case-control study. Transfusion. 2018;58:498-506.

32. Inaba K, Branco BC, Rhee P, Blackbourne LH, Holcomb JB, Teixeira PG, et al. Impact of plasma transfusion in trauma patients who do not require massive transfusion. J Am Coll Surg. 2010;210:957-65.

33. Narick C, Triulzi DJ, Yazer MH. Transfusion-associated circulatory overload after plasma transfusion. Transfusion. 2012;52:160-5.

34. Innerhofer P, Fries D, Mittermayr M, Innerhofer N, von Langen D, Hell T, et al. Reversal of traumainduced coagulopathy using first-line coagulation factor concentrates or fresh frozen plasma (RETIC): a single-centre, parallel-group, open-label, randomised trial. Lancet Haematol. 2017;4:e258e71.

35. Barry M, Trivedi A, Miyazawa BY, Vivona LR, Khakoo M, Zhang H, et al. Cryoprecipitate attenuates the endotheliopathy of trauma in mice subjected to hemorrhagic shock and trauma. J Trauma Acute Care Surg. 2021;90:1022-31.

36. Wu F, Chipman A, Pati S, Miyasawa B, Corash L, Kozar RA. Resuscitative Strategies to Modulate the Endotheliopathy of Trauma: From Cell to Patient. Shock. 2020;53:575-84.

37. Jensen $\mathrm{NH}$, Stensballe J, Afshari A. Comparing efficacy and safety of fibrinogen concentrate to cryoprecipitate in bleeding patients: a systematic review. Acta Anaesthesiol Scand. 2016;60:103342.

38. Morrow GB, Carlier MSA, Dasgupta S, Craigen FB, Mutch NJ, Curry N. Fibrinogen Replacement Therapy for Traumatic Coagulopathy: Does the Fibrinogen Source Matter? Int J Mol Sci. 2021;22:2185.

39. Johansson PI, Sørensen AM, Perner A, Welling KL, Wanscher M, Larsen CF, et al. Disseminated intravascular coagulation or acute coagulopathy of trauma shock early after trauma? An observational study. Crit Care. 2011;15:R272.

40. Wong $\mathrm{H}$, Curry $\mathrm{N}$. Do we need cryoprecipitate in the era of fibrinogen concentrate and other specific factor replacement options? Vox Sang. doi: 10.1111/voxs.12376.

41. Committee on Trauma of the American College of Surgeons. ACS TQIP Massive Transfusion in Trauma Guidelines. Available at: https://www.facs.org/-/media/files/qualityprograms/trauma/tqip/transfusion_guildelines.ashx. Accessed June 14, 2021.

\section{Figures}




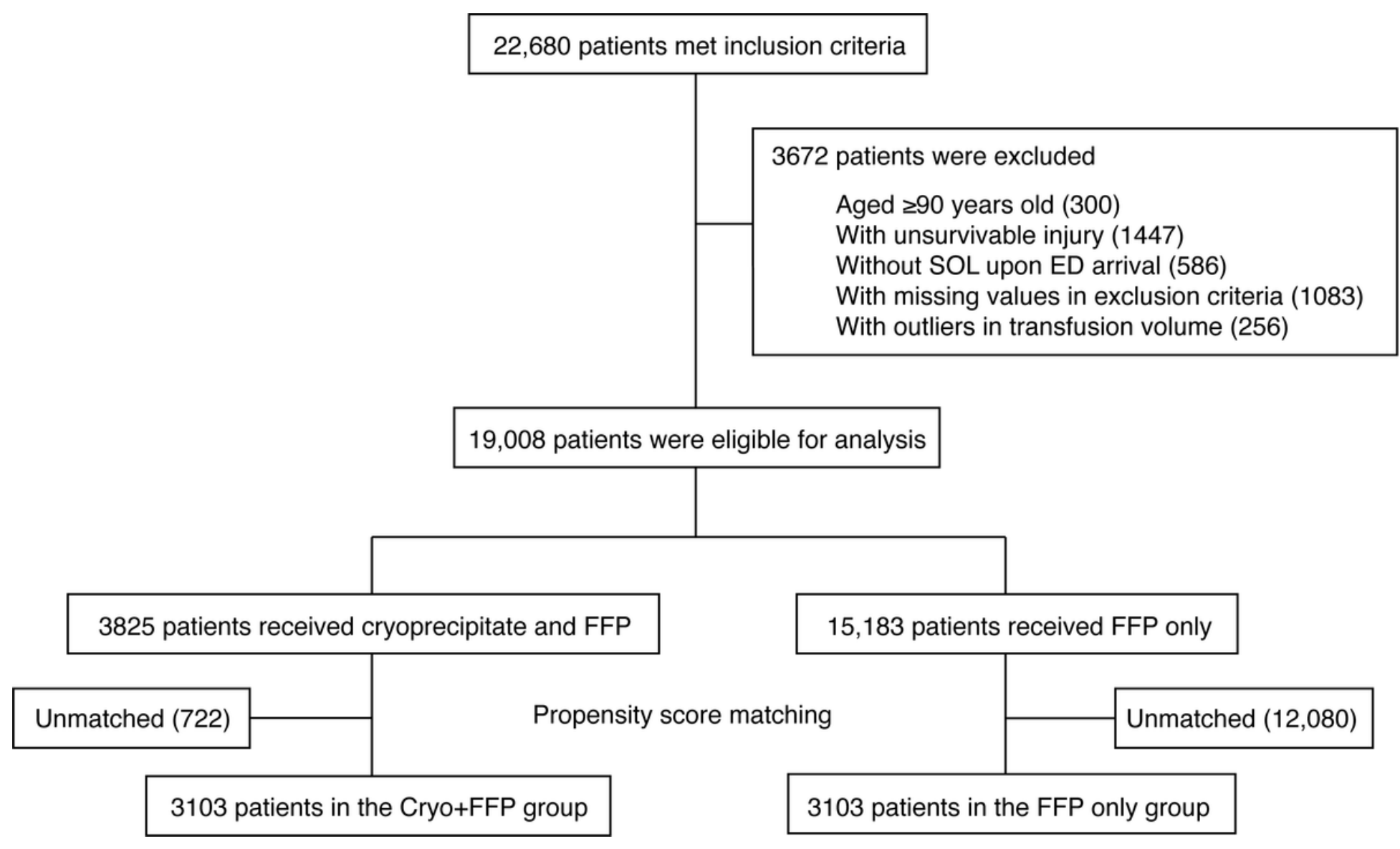

\section{Figure 1}

Flow diagram of patient selection Abbreviations: SOL, signs of life; ED, emergency department; FFP, fresh frozen plasma; Cryo, cryoprecipitate. 


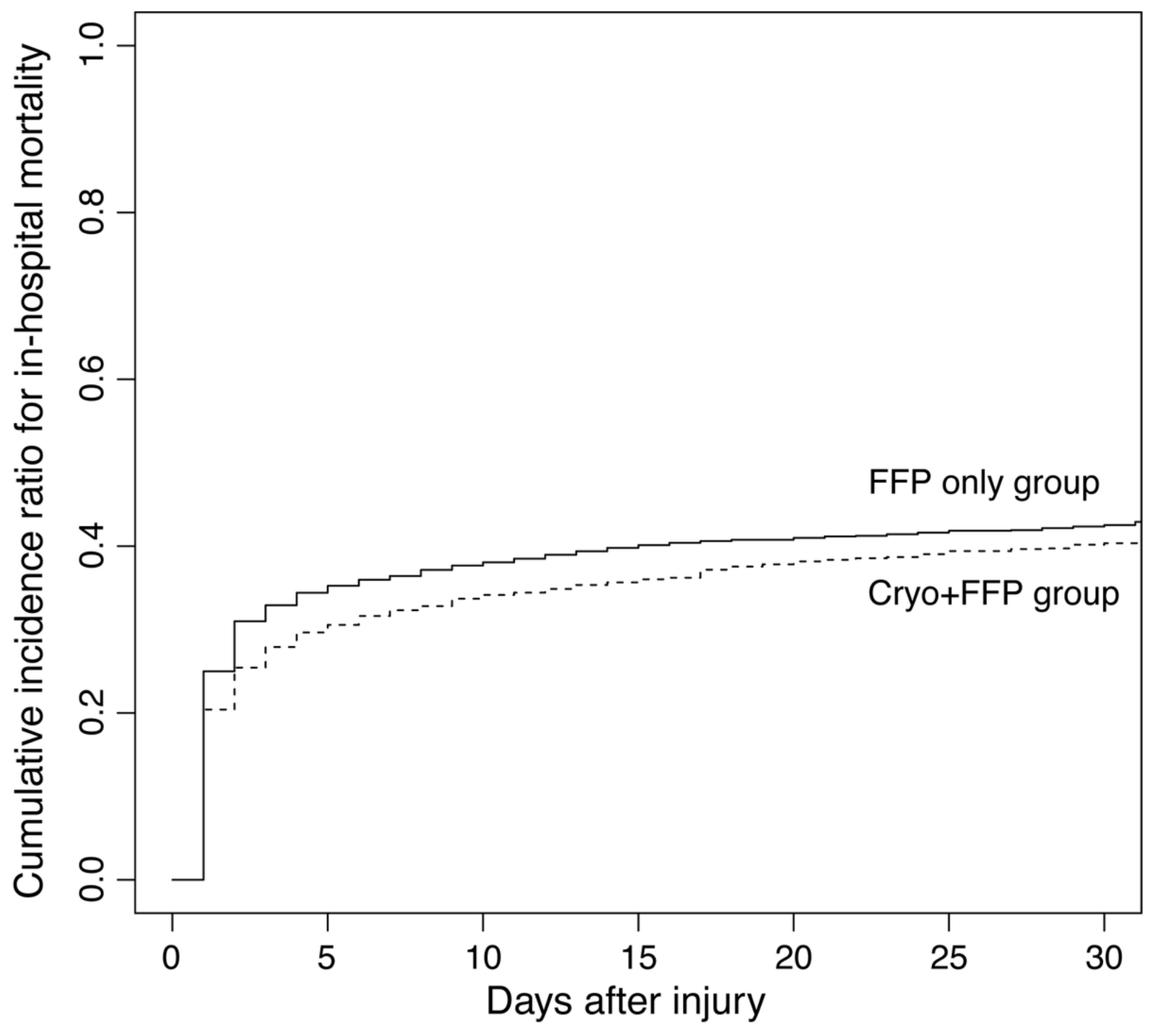

Figure 2

Cumulative incidence curves for in-hospital mortality in the propensity score-matched subjects Abbreviations: FFP, fresh frozen plasma; Cryo, cryoprecipitate.

\section{Supplementary Files}

This is a list of supplementary files associated with this preprint. Click to download.

- TableS1.docx

- Tables2.docx 
- TableS3.docx

- TableS4.docx

- Tables5.docx 